\title{
BMJ Open Health system readiness for non- communicable diseases at the primary care level: a systematic review
}

\author{
Ashraful Kabir (D) , ${ }^{1}$ Md Nazmul Karim, ${ }^{1}$ Rakibul M Islam (D) , ${ }^{2}$ Lorena Romero, ${ }^{3}$ \\ Baki Billah $^{1}$
}

To cite: Kabir A, Karim MN, Islam RM, et al. Health system readiness for noncommunicable diseases at the primary care level: a systematic review. BMJ Open 2022;12:e060387. doi:10.1136/ bmjopen-2021-060387

- Prepublication history and additional supplemental material for this paper are available online. To view these files, please visit the journal online (http://dx.doi.org/10.1136/ bmjopen-2021-060387)

Received 21 December 2021 Accepted 26 January 2022

D) Check for updates

(c) Author(s) (or their employer(s)) 2022. Re-use permitted under CC BY-NC. No commercial re-use. See rights and permissions. Published by BMJ.

${ }^{1}$ School of Public Health and Preventive Medicine, Monash University, Melbourne, Victoria, Australia

${ }^{2}$ Women's Health Research Program, Monash University, Melbourne, Victoria, Australia

${ }^{3}$ The lan Potter Library, Ground

Floor, AMREP Building, The

Alfred, Melbourne, Victoria, Australia

Correspondence to

Ashraful Kabir;

md.kabir@monash.edu

\section{ABSTRACT}

Objective To synthesise evidence on the primary healthcare system's readiness for preventing and managing non-communicable diseases (NCDs).

Design Systematic review.

Data sources Ovid MEDLINE, EMBASE, CINAHL, PsycINF0 and Scopus were searched from 1 January 1984 to 30 July 2021, with hand-searching references and expert advice.

Eligibility criteria Any English-language health research with evidence of readiness/preparedness of the health system at the primary healthcare level in the context of four major NCDs: diabetes mellitus, cancer, chronic respiratory diseases (CRDs) and cardiovascular diseases (CVDs).

Data extraction and synthesis Two authors independently extracted data and assessed the bias. The full-text selected articles were then assessed using the Mixed Methods Appraisal Tool. Health system readiness was descriptively and thematically synthesised in line with the health system dynamics framework.

Results Out of 7843 records, 23 papers were included in this review (15 quantitative, 3 qualitative and 5 mixedmethod studies). The findings showed that existing literature predominantly examined health system readiness from the supply-side perspective as embedded in the WHO's health system framework. However, at the primary healthcare level, these components are insufficiently prepared for NCDs. Among NCDs, higher levels of readiness were reported for diabetes mellitus and hypertension in comparison to CRDs (asthma, chronic obstructive pulmonary disease), CVDs and cancer. There has been a dearth of research on the demand-side perspective, which is an essential component of a health system and must be addressed in the future research. Conclusion The supply-side components at the primary healthcare level are inadequately ready to address the growing NCD burden. Improving supply-side factors, with a particular focus on CRDs, CVDs and cancer, and improving understanding of the demand-side components of the health system's readiness, may help to prevent and manage NCDs at the primary healthcare level.

\section{INTRODUCTION}

Globally, non-communicable diseases (NCDs) are the leading causes of deaths and disabilities, accounting for 41 million

\section{Strengths and limitations of this study}

Data synthesis was informed by the health system dynamics framework, which offers a deeper and more comprehensive (both supply-side and demand-side factors) understanding of primary healthcare system readiness for non-communicable diseases.

- We conducted an extensive systematic search of literature with hand-searching references and expert advice regarding health system readiness for noncommunicable diseases at the primary care level, which increases the validity and trustworthiness of this review's findings.

- Meta-analysis was not possible due to heterogeneity of study designs, methods and techniques, as well as the studies' focus on a variety of health system components.

- A few studies that reported health system readiness at combined primary and secondary healthcare levels were excluded.

deaths (71\% of all deaths) annually, ${ }^{1}$ with $77 \%$ occurring in low-income and middleincome countries (LMICs). ${ }^{12}$ The current increased NCD burden may be due to the rise of the ageing population, rapid and/or unplanned urbanisation and lifestyle-related factors (eg, physical inactivity, unhealthy diets and consumption of tobacco products and alcohol). ${ }^{3}$ If current trends continue, the estimated cumulative deaths from NCDs will reach 52 million by $2030,{ }^{3}$ and NCDrelated cost was projected to be US $\$ 47$ trillion between 2010 and 2030. ${ }^{4}$ NCDs' predicted health outcomes and economic burden will have adverse consequences, such as prolonged illness or disability, greater treatment costs, loss of productivity and substantial opportunity cost, which will eventually affect households' economy and well-being. ${ }^{45}$ The impact of NCDs may result in increased poverty, higher inequality and low quality of life. Considering the immense influence of NCDs, many commitments and control 
strategies have been made at the global, national and local levels to prevent and manage them. ${ }^{6-8}$ The Sustainable Development Goals, for example, by 2030, targeted one-third reduction of premature deaths from the four major NCDs of diabetes mellitus (DM), cancer, chronic respiratory diseases (CRDs) and cardiovascular diseases (CVDs) ${ }^{89}$ among people aged 30-69.

Primary healthcare is crucial for promoting essential healthcare services and achieving improved health outcomes, particularly in countries with resource-poor settings. ${ }^{3}{ }^{10-12}$ Growing evidence shows that a wellfunctioning primary healthcare system has immense potential for improving global health outcomes due to its extensive coverage, cost-effectiveness, well-structured network of healthcare facilities, affordable technologies, socially and culturally acceptable intervention methods and broad community participation. ${ }^{10} 1314$ NCD prevention and management differ from that of acute conditions, where the primary healthcare approach has a powerful impact. Unlike acute conditions, NCD prevention and management require extended or even life-long healthcare support, early case detection, psychosocial promotion, risk factor identification, self-management, behavioural modifications and regular medical support, such as adherence to medical procedures and treatment. ${ }^{3}$ The primary healthcare system is typically the first-line contact for individuals seeking care, making it easier for patients to continue follow-up contacts. ${ }^{15}$ Therefore, it can be viewed as the most effective and appropriate mechanism for addressing NCDs.

While the literature emphasises the roles and importance of the primary healthcare system in preventing and managing NCDs following a dozen of global commitments and strategies, little is known about the extent to which it is ready to deliver NCD services. ${ }^{16}{ }^{17}$ The concept of 'health system readiness' is often explained in terms of the health system 'components' or 'framework'. Until recently, health system readiness was mostly defined and presented in the context of the WHO's health system framework, proposed in 2008, which described six 'key elements' or 'building blocks': health service delivery (HSD), health workforce, health financing (HF), health information system (HIS), leadership and governance (L\&G), medical products, knowledge and technologies (MPK\&T). ${ }^{18}$ However, the WHO's model is viewed as having limited capacity to comprehensively explain how and whether different health system elements within a broader societal context interact and are influenced, as well as how population/individual behaviour and choices and the process impact this mechanism. ${ }^{19}{ }^{20}$ In order to provide an exhaustive understanding of system interactions, van Olmen et al proposed the 'health system dynamics framework', which included the WHO's six building blocks and concurrent literature. It is comprised of 10 elements that analyse their interactions and functions under a broader societal context. ${ }^{21}$

Guided by the 'health system dynamics framework', this systematic review aimed to synthesise the current evidences on primary healthcare system readiness and evaluate its response to NCDs on a global scale. The findings of this review will help policymakers, public health planners and researchers focus on the further actions required to establish a well-prepared health system at the primary healthcare level to address the growing NCD burden.

\section{METHODS}

\section{Protocol and registration}

This review was conducted following the Preferred Reporting Items for Systematic Reviews and Meta-Analysis guideline ${ }^{22}$ and was registered on the Research Registry (REVIEWREGISTRY1163).

\section{Inclusion criteria}

This review included studies that reported on the readiness/preparedness of various health system components at the primary healthcare level in the context of four major NCDs: DM, cancer, CRDs and CVDs. Where studies reported health system preparedness at the primary and secondary care level combined, only information related to the primary healthcare level was included. However, studies in which the primary and secondary care level data could not be separated were excluded.

\section{Exclusion criteria}

Studies on other NCDs such as arsenicosis, kidney diseases, mental health disorders, hearing disability, oral disease, birth defects and road injuries were excluded. Papers that focussed on NCD interventions/programmes beyond the primary healthcare level were likewise excepted. Editorials, letters, opinion articles, narrative or systematic reviews, study protocols, conference abstracts, posters, reports and book chapters were also not considered. Additionally, works that were published in a language other than English were excluded.

\section{Data sources and search strategy}

The search strategy aimed to find English-language studies in five databases (Ovid Medline, Ovid Embase, Ovid PsycInfo, CINAHL and Scopus) published between 1 January 1984 and 30 July 2021 (figure 1). The WHO's health system model proposed in 1984 was considered appropriate to identify and assess the key components of the primary healthcare system. The studies published in 1984 onward were deemed to be relevant to this review. Therefore, the earliest date of the search was set to ensure the optimum number of studies published since 1984. The search strategies used a combination of subject headings and free-text terms that aimed to cover the areas of ${ }^{1}$ NCDs (eg, chronic disease or chronic conditions or chronic disorders), and ${ }^{2}$ primary health system (eg, primary health service or first-level healthcare facility or local health system or local-level health facility) $\mathrm{and}^{3}$ readiness or preparedness or capacity. 


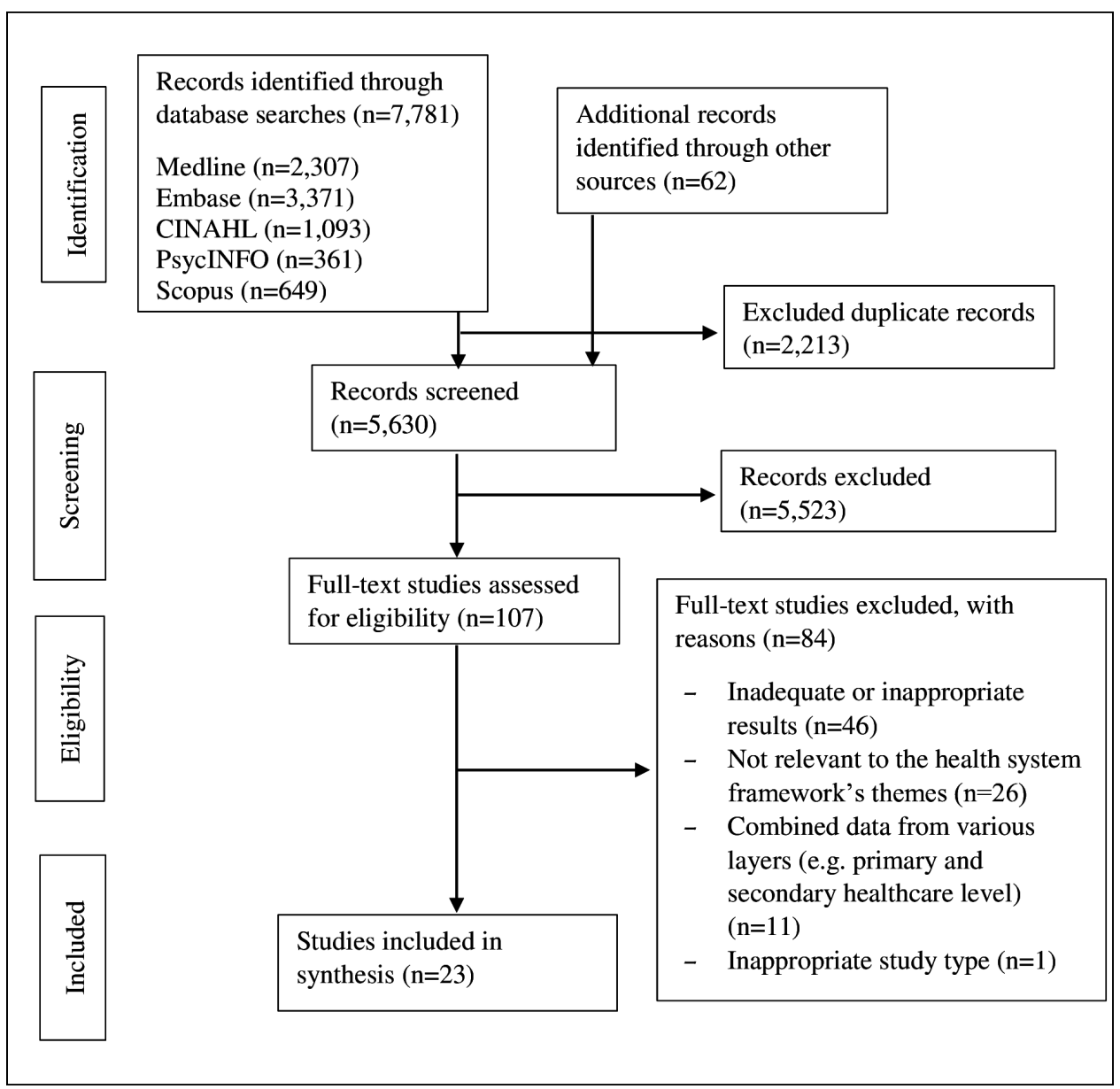

Figure 1 Preferred Reporting Items for Systematic Reviews and Meta-Analysis flowchart for study inclusion.

Searches were adapted as appropriate to the specifications of each of the five databases. The final searches are presented in the online supplemental appendix 1 . Handsearching and reference checking of citations and reference lists were undertaken, and additional records were identified through personal consultations with experts, including researchers, administrators, policy planners and public health practitioners.

\section{Data extraction}

Three authors were involved in the data extraction process. First, records identified through database and manual searches were imported into the Endnote library (EndNote X9.2, Thomson Reuters 2019). Afterwards, the duplicate records were removed. Next, two authors (AK and MNK) independently screened the studies based on their titles and/or abstracts. The full-text selected articles were then assessed using the inclusion and exclusion criteria and the standard quality assessment. When inconsistencies and discrepancies arose, a senior author (BB) was brought in to resolve the disagreements through discussion and consensus. A standardised data extraction sheet was developed and piloted. The extraction sheet contains the following study-specific information: authors, publication year, country, study aims, study design and settings, sample size and participants, data collection method and tool used, NCD/risk factor studied, health system component focus and key findings.

\section{Quality assessment}

The Mixed Methods Appraisal Tool (MMAT) was used to assess the methodological quality of the included studies. ${ }^{23}$ The distribution of MMAT scores varied with the study design and the evaluation of some selected parameters. The score is $25 \%$ when quantitative (QUAN) study=1, qualitative (QUAL) study=1 or mixed-method (MM) study $=0$. It is $50 \%$ when $\mathrm{QUAN}=2$, QUAL $=2$ or $\mathrm{MM}=1 ; 75 \%$ when $\mathrm{QUAN}=3$, QUAL $=3$ or $\mathrm{MM}=2$; and it is $100 \%$ when $\mathrm{QUAN}=4$, $\mathrm{QUAL}=4$ or $\mathrm{MM}=3$. Thus, each study was given a score ranging from $25 \%$ to $100 \%$ (table 1). Two authors (AK and MNK) independently assessed the studies' quality, and the senior author (BB) cross-checked them. Discrepancies and disagreements were resolved through discussion.

\section{Data synthesis}

Data analysis was guided by the health system dynamics framework. ${ }^{24}$ The following themes were synthesised using this framework: (1) HSD, (2) healthcare workforce, (3) HF, (4) access to MPK\&T, (5) HIS, (6) L\&G and (7) community perspective. Under these themes, data from quantitative studies were reported descriptively using 
Table 1 Type of research design and associated quality of included studies $(n=23)$

\begin{tabular}{llllll}
\hline & \multirow{2}{*}{$\begin{array}{l}\text { Number of } \\
\text { Study design }\end{array}$} & \multicolumn{5}{c}{ MMAT score (\%) } \\
\cline { 3 - 6 } & studies (\%) & $\mathbf{2 5}$ & $\mathbf{5 0}$ & $\mathbf{7 5}$ & $\mathbf{1 0 0}$ \\
\hline Quantitative & $15(65)$ & - & 5 & 7 & 3 \\
Qualitative & $3(13)$ & - & 1 & 2 & - \\
Mixed-methods & $5(22)$ & 1 & 2 & 2 & - \\
\hline
\end{tabular}

Note: entries in the table show the number of studies. MMAT, Mixed Methods Appraisal Tool.

frequencies or percentages, while qualitative studies were synthesised by determining themes. In this process, a few steps were followed: (1) familiarising, (2) identifying themes (health system components), (3) indexing, (4) charting and (5) mapping and interpreting. Data from mixed-methods studies were analysed both descriptively and thematically analysed. The heterogeneous study design of the included studies precluded a meaningful meta-analysis in this review.

\section{Patient and public involvement}

There was no patient or public involvement.

\section{RESULTS}

\section{General characteristics of the study}

Initially, 7843 studies were retrieved, from which 2213 duplicates were excluded (figure 1). Then, 5630 studies were excluded based on a title and abstract review, with 107 meeting the inclusion criteria for a full-text review. Following the full-text review, 23 studies were ultimately included in this study (table 2): 15 were quantitative (cross-sectional), ${ }^{25-39} 3$ were qualitative ${ }^{40-42}$ and 5 were mixed-method studies. ${ }^{43-47}$ Most of the research was conducted in resource-poor settings (20 studies), mostly in sub-Saharan Africa and South Asian countries. Eighteen studies focussed on the HSD component at the primary healthcare level, while four studies addressed the L\&G (figure 2A). Eight studies were conducted in the South Asian-East Asia Region, and only a single study $(n=1)$ was performed in both the Region of the Americas and the European Region. One study involved multiple nations (Benin, Eritrea, Sudan, Syria, Bhutan, Sri Lanka, Vietnam and Suriname) (figure 2B). DM was the most studied NCDs, with 12 studies reported on it, while mental illness was the least researched, with only two studies (figure 2C) focussed on it. Thirteen studies addressed multiple NCDs, six focussed on a single NCD and four did not mention any specific NCD (eg, termed chronic diseases) (figure 2D).

\section{Health service delivery}

Of the 23 studies, 18 addressed issues related to the HSD system's readiness in preventing and managing NCDs at the primary healthcare level. Eleven of the 18 studies were quantitative studies, assessing primary healthcare facilities' readiness in implementing the WHO SARA reference manual ${ }^{25-27} 2930363845$ or WHO PEN interventions. ${ }^{33-35}$ Three papers adopted the qualitative approach, ${ }^{40-42}$ while another three used the mixedmethod approach. ${ }^{43} 4547$ Four studies focussed on a single NCD: DM, $\mathrm{CVD}^{40}$ or hypertension (HTN) ${ }^{26}{ }^{32}$ Five papers studied two NCDs, ${ }^{25} 35363843$ while seven investigated multiple NCDs and risk factors. ${ }^{27} 303139414248$ However, two articles did not specify the NCDs that were evaluated. ${ }^{347}$ Most of the studies found that healthcare facilities had insufficient capacity to deliver NCD prevention, care and treatment at the primary level. Among the NCDs, a higher level of readiness at the primary healthcare level was reported for HTN prevention and management. The availability of HTN services at healthcare facilities was reported to be $92.9 \%$ in Uganda $^{32}$ and $86 \%$ in Tanzania ${ }^{25}$; however, one study found that HTN preparedness was only $28 \%$ in Tanzania's outpatient care. ${ }^{26}$ A mixed-methods study in Thailand revealed that commune health station were significantly prepared to manage HTN. ${ }^{44}$ The services readiness for CVD (47.8\%), and DM (50\%), were reported at the Upazila Health Complex (UHC) in 2014 in Bangladesh. ${ }^{29}{ }^{38}$ However, the most recent data reported the availability of services largely varied from Community Clinic (CC) to 'UHC' for cervical cancer $(0.4 \%-37.5 \%)$, CRD $(34.1 \%-93.9 \%)$, CVD (1.4\%-69.6\%), DM (0.9\%-84.5\%) and HTN $(3.5 \%-91.5 \%){ }^{39}$ In Vietnam, only $25 \%$ of commune health centres were equipped to prevent, diagnose and treat major NCDs, with a noticeably lower utilisation rate of services by the users. ${ }^{27}$ Capacity for managing DM was predominantly low across all studies; however, one study in Tanzania ${ }^{25}$ found that care for DM was available in $79 \%$ of healthcare facilities. Moreover, a lower level of readiness for managing CVD was reported across countries. ${ }^{31404245}$ Qualitative studies conducted in Thailand ${ }^{40}$ and India ${ }^{43}$ noted facilities' low-level preparedness to manage HTN, DM and CVD, with healthcare facilities/ programmes lacking effective community engagements and limited support from the national programmes. In Kien $e$ t $a l$ s 2018 study conducted in Vietnam, one of the district-level health staff shared the following:

[In our district] we implemented the hypertension programme for only four communes and implemented the diabetes programme for four other communes [among 18 communes]. We do not have any NCD programmes for the rest of the communes. ${ }^{41}$

In a cross-sectional study conducted in Madhya Pradesh, India, the preparedness level for DM and HTN was reported to be slightly high. ${ }^{35}$ However, inadequate capacity was found for managing the common NCDs in a qualitative study in Odisha and Kerala, India. ${ }^{42}$ Lower levels of readiness for major NCDs have also been commonly reported in Zambia ${ }^{33}$ and Ghana. ${ }^{34}$

Overall, the delivery of NCD services was affected by multiple factors and revealed to be insufficient at the primary healthcare level. Inadequate and ill-equipped 


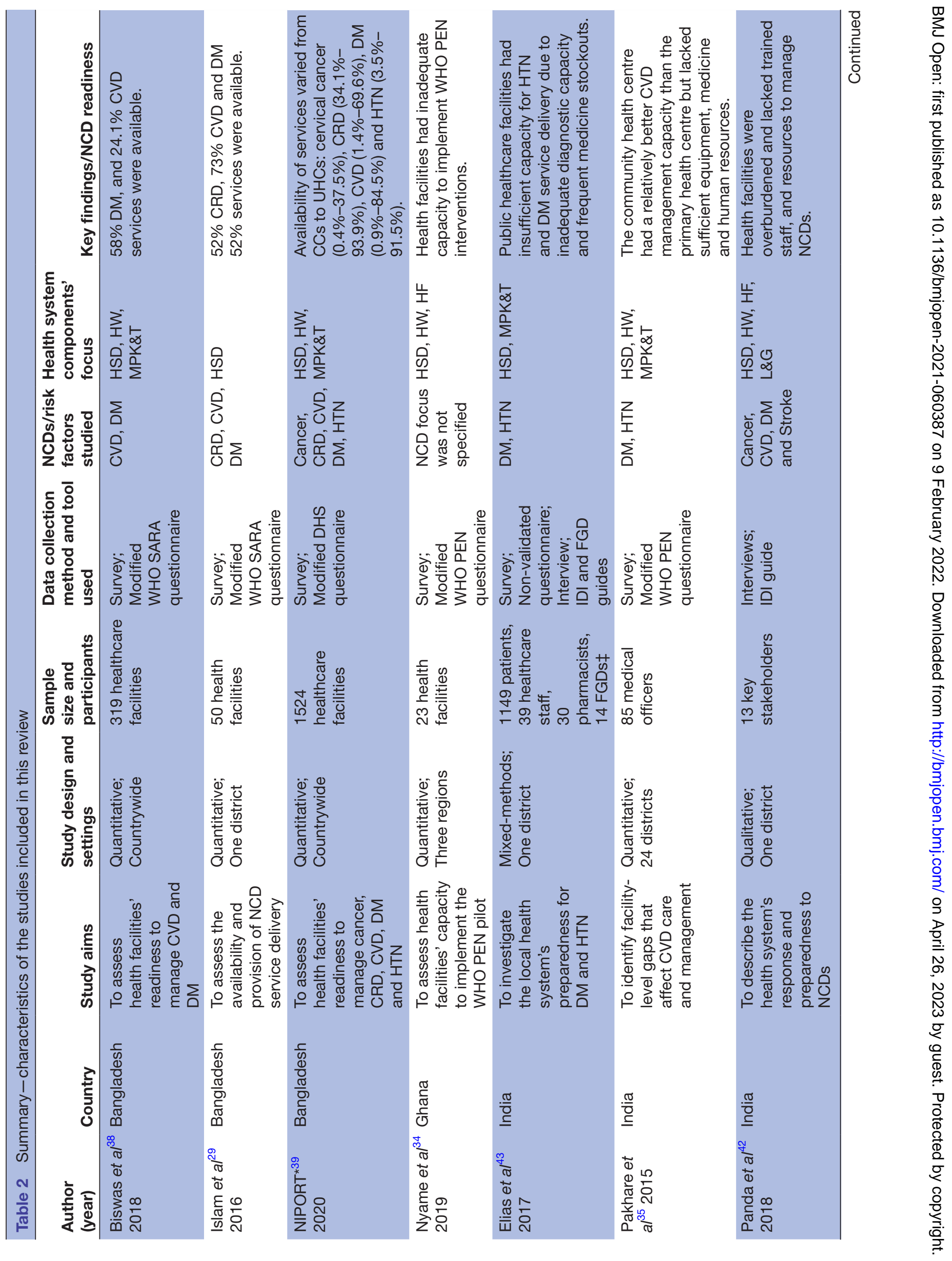




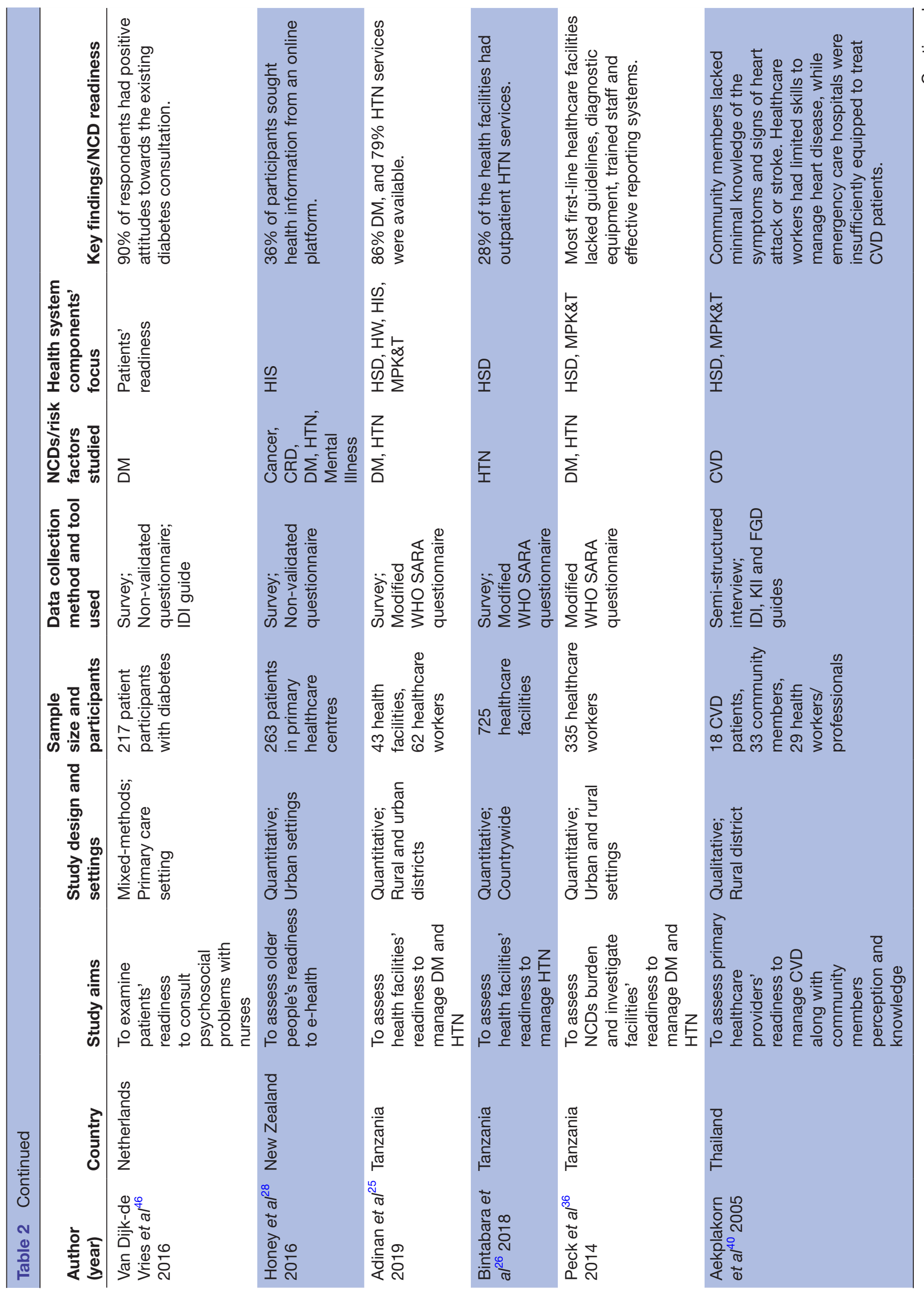




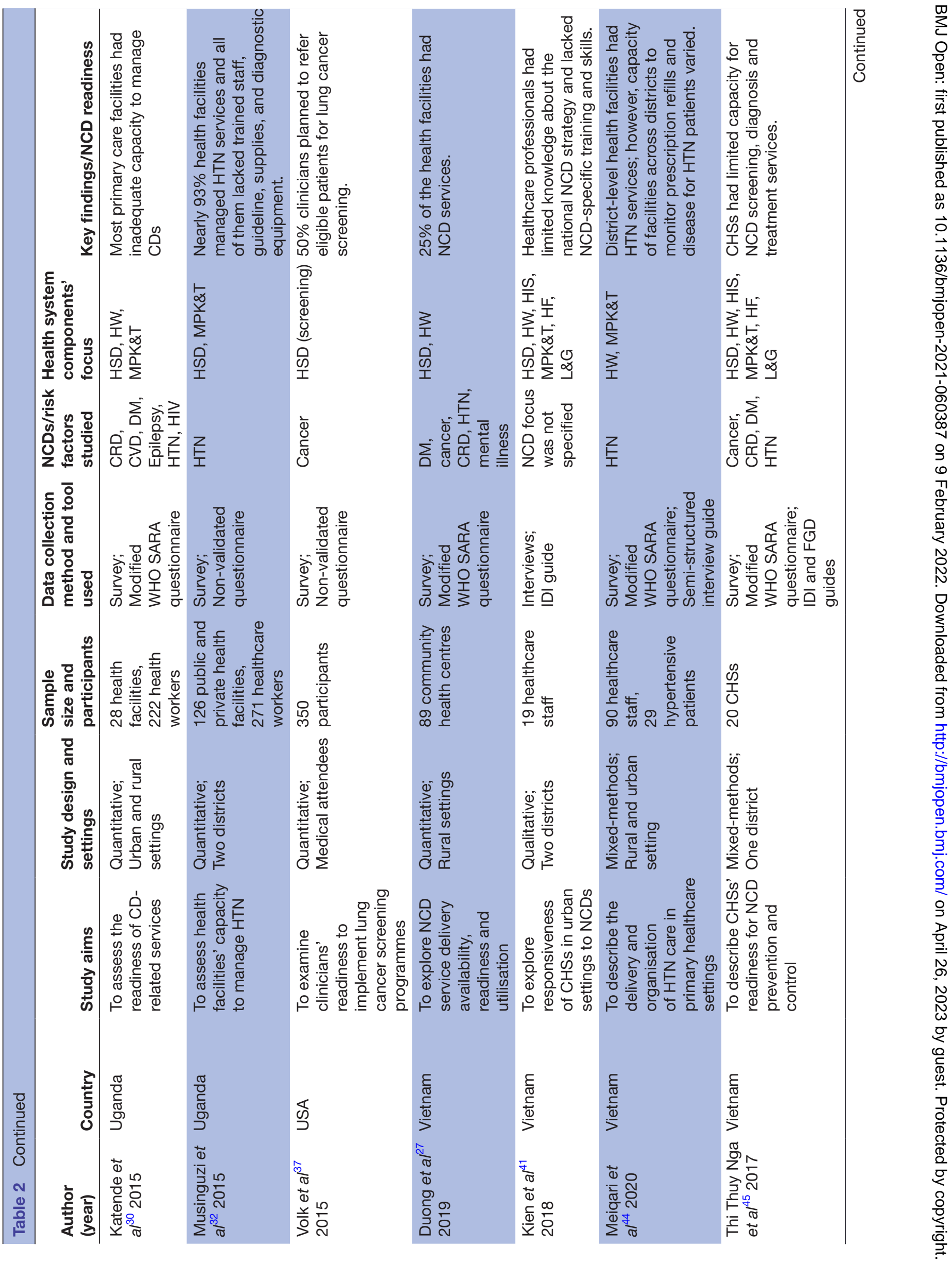



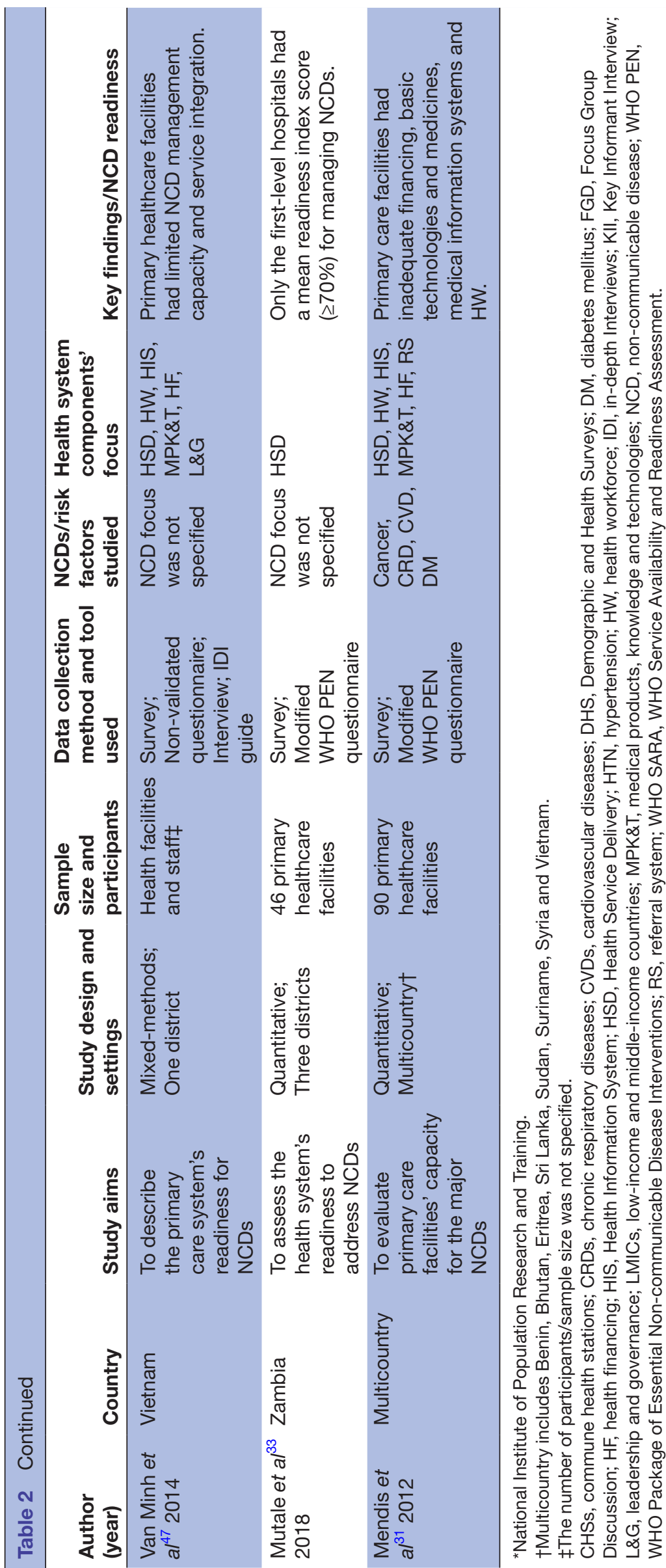
A

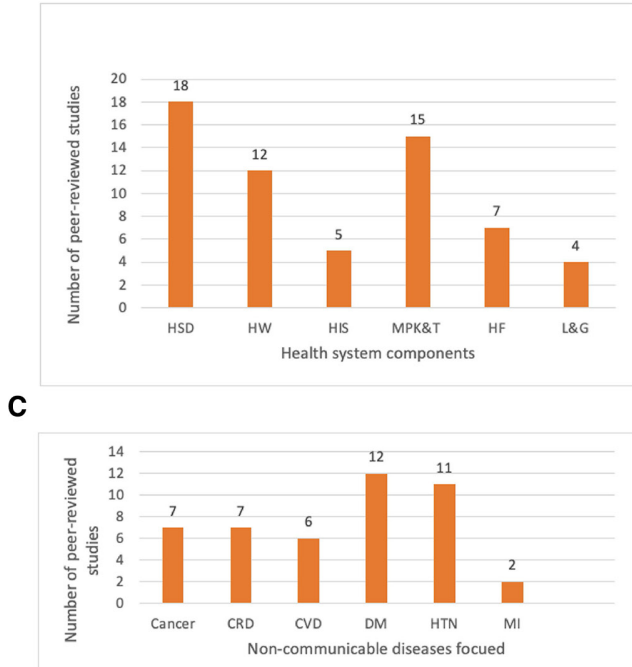

B

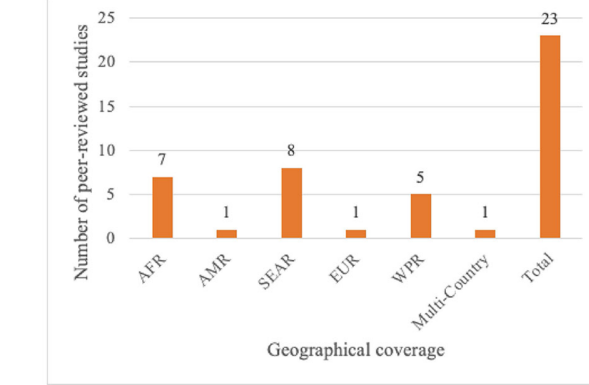

D

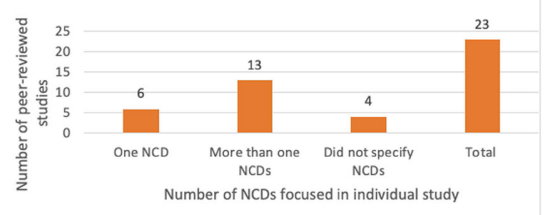

Figure 2 Number of published studies that investigated the primary healthcare system's readiness between January 1984 and July 2021, broken down by NCD type, NCD focus and WHO region. AFR, African Region; AMR, Region of the Americas; CRD, chronic respiratory diseases; CVD, cardiovascular diseases; DM, diabetes mellitus; EUR, European Region; HIS, health information system; HF, health financing; HSD, health service delivery; HTN, hypertension; HW, health workforce; L\&G, leadership and governance; MI, mental Illness; MPK\&T, medical products, knowledge and technologies; NCDs, noncommunicable diseasesSEAR, South East-Asian Region; WPR, Western Pacific Region.

healthcare facilities were the most common issues hampering service delivery. ${ }^{25} 2731-3543$ Moreover, notable key barriers include patients' lack of self-management education and knowledge, ${ }^{25}$ primary-level healthcare professionals' limited NCD management skills and national NCD strategies, ${ }^{25} 41$ insufficient NCD service management and implementation capacity of locallevel healthcare organisations, ${ }^{26} 47$ a weak referral and follow-up system, ${ }^{30} 31$ poor adherence to clinical guidelines, ${ }^{25} 303236$ inadequate screening opportunity, ${ }^{45}$ lack of information-education-community material ${ }^{45}$ and the healthcare facility's rural location.

\section{Healthcare workforce}

Twelve of the studies reviewed reported a healthcare workforce issue related to NCD services and care. According to these papers, a common bottleneck for NCD services is insufficient primary-level healthcare professionals. One cross-sectional study in Tanzania reported only $53 \%$ and $15 \%$ of healthcare facilities had trained health professionals to manage HTN and DM, respectively. ${ }^{25}$ In Thailand ${ }^{40}$ and Vietnam, ${ }^{45}{ }^{47}$ there was an acute lack of trained healthcare staff to manage CVD. Moreover, a study conducted in Uganda found that only $26 \%$ and $16 \%$ of primary healthcare staff had an adequate level of knowledge to manage DM and HTN outpatients, respectively. ${ }^{30}$ This study also revealed that medical doctors had a higher level of knowledge (85\% for HTN and $8 \%$ for DM) than nurses (8\% for HTN and $4 \%$ for DM) ${ }^{30}$ One study in Vietnam reported that only $9 \%$ of primary healthcare facilities in rural and urban locations had five categories of human resources (medical doctor, assistant doctor, nurse, midwife and pharmacist) to deliver HTN services. ${ }^{44}$ The shortage of trained healthcare staff (at least one staff received in-service training in the last 24 months before the data collection date) was reported at the primary healthcare in Bangladesh. ${ }^{39}$ The trained staff for cervical cancer (29\% trained staff at the UHCs, but no trained staff in CCs and union-level facilities), CRD (4\% union-level facilities, 11\% CCs and 29\% UHCs), CVD (7\% union-level facilities, 15\% CCs and 40\% UHCs), DM (3\% union-level facilities, $14 \%$ CCs and 28\% UHCs) and HTN (6\% union-level facilities, 10\% CCs and 39\% UHCs) were reported.$^{39}$ According to a multicountry study, physicians at primary healthcare facilities were only available in two of the eight participating nations, while nurses and healthcare assistants were the key professionals for NCD services in the remaining six countries. ${ }^{31}$ A study in Ghana found that more than half of the healthcare centres lacked at least one medical doctor and nurse trained in NCDs. ${ }^{34}$ In India, while two medical officers were available on average at community health centres to manage DM, CVD, HTN and cancer, this number was lowest (less than half) in primary healthcare centres. ${ }^{35}$ In qualitative studies conducted in India ${ }^{42}$ and Vietnam, ${ }^{41}$ insufficient healthcare staff jeopardised NCD services in primary care facilities. An NCD programme officer in Odisha, India and a national-level health worker in Vietnam shared their respective thoughts:

In a big community health centre like ours, there should be more health workforce, and there should be a special training programme for all the health workers. ${ }^{42}$

For the health workforce at commune health stations, some facilities lack human resources and/or 
capacity. They must be strengthened in their capacity to provide services for NCD prevention, consultation, early detection and management. The reason for this is that we have not implemented NCD services systematically at primary healthcare facilities. ${ }^{41}$

\section{Health financing}

Seven studies found that inadequate funding/budget support from the national healthcare programme compromised effective NCD service and care at the primary healthcare level. Furthermore, the absence or limitation of healthcare insurance coverage jeopardised NCD services and care. One study in India reported that less than $3 \%$ of households had insurance coverage ${ }^{43} \mathrm{~A}$ study in Ghana revealed that healthcare financing is organised by the government as the 'National Health Insurance Scheme', and only those who paid the premium received its benefits. ${ }^{34}$ Limited public financial/budgetary support has also been identified as a major barrier to NCD services in primary healthcare in Vietnam. ${ }^{45} 47$ A national-level health worker in Vietnam conveyed the following to Kien et al in 2018:

The budget for NCD primary health care services is extremely limited; [funding is] mainly through national target programmes on NCDs, but the programmes have been reduced. There are some barriers to health insurance reimbursement for NCDs at the primary health care level. ${ }^{41}$

Similarly, in a qualitative study, a medical officer from Odisha, India shared his observation:

Since there is no existing system, funds do not reach the grassroots level. There is no funding. ${ }^{42}$

\section{Access to medical products, knowledge and technologies}

Across countries and regions, a lack of supply-side factors, such as MPK\&T to prevent and manage NCDs, has been widely reported. Fifteen studies reported inadequate or interrupted access to supplies and technologies at the primary healthcare level, which are vital for diagnosing and treating NCDs. In Bangladesh, the availability of medicine widely varied at the UHCs based on their types for DM (metformin: 38.1\%, glibenclamide: 7.4\%), CRD (salbutamol: 91.6\%, epinephrine: 0.3\%), CVD (amiodipine/nifedipine: 41.5\%, aspirin: 2.6\%), and HTN (amlodipine/nifedipine: 44.7\%, thiazide: $1.4 \%$ ), but no supply in the CCs were reported. ${ }^{39}$ In India, the essential drugs for the management of HTN (beta-blockers and calcium channel blockers) were available at most of the primary health centres (PHCs) and community health centres; however, other drugs (except metformin) were largely unavailable across facilities that resulted in $90 \%$ of patients with NCD in India to rely on private providers/facilities for NCD service and care. ${ }^{35}$ More than $60 \%$ of PHC-level facilities faced a shortage of essential DM medicine, with over $30 \%$ of PHCs having a medicine stockout of more than 6 months. Only $38 \%$ of PHCs had functional laboratory facilities. ${ }^{43}$ According to a study conducted in Tanzania, $50 \%$ of health centres, $24 \%$ of dispensaries and $80 \%$ of hospitals had HTN and DM medicines in hand; however, more than one-third of these locations lacked basic laboratory facilities. ${ }^{25} \mathrm{~A}$ qualitative study in Vietnam ${ }^{41}$ and a qualitative multicountry investigation (Benin, Bhutan, Eritrea, Sri Lanka, Sudan, Suriname, Syria and Vietnam) ${ }^{31}$ likewise reported the shortage of medicine and basic diagnostic facilities at primary healthcare facilities. Moreover, basic amenities and equipment for NCDs were in short supply in Ugandan healthcare facilities (hospitals and healthcare centres), with more than half of them lacking the recommended antihypertensive drug and nearly $30 \%$ lacking a blood pressure device. ${ }^{32}$ Likewise, Tanzanian healthcare facilities reported a shortage of the recommended medicine and supplies required for HTN and DM service and care. ${ }^{36}$ Similarly, a mixed-method study found a scarcity of medical products and equipment for CRD, DM, cancer and HTN in Vietnam. ${ }^{45}$ However, basic equipment and diagnostic facilities such as stethoscope $(93.2 \%$ CCs, 96.9\% UHCs), blood pressure apparatus $(85.6 \%$ CCs, 95.4\% UHCs), adult scale (90.9\% CCs, $82.9 \%$ UHCs), blood glucose testing (22.2\% CCs, $48.9 \%$ UHCs), urine protein (0\% CCs, 36.2\% UHCs) and urine glucose $(0 \%$ CCs, $30.4 \%$ UHCs) were available in Bangladesh. ${ }^{39}$

\section{Health information system}

Studies that assessed the HISs' readiness were limited. Only five papers addressed the HIS required for optimising NCD care at the primary healthcare level. ${ }^{25} 31414547$ These studies extensively reported on weak HISs for detecting, treating and monitoring patients with NCD in primary healthcare settings. Furthermore, only $52.9 \%$ of primary healthcare facilities in Tanzania were prepared to collect, analyse and use local-level data for HTN and DM services. ${ }^{25}$ According to a multicountry survey, $85 \%$ of healthcare facilities created paper-based (patient register) individual-level information for patients who attended the facilities, but only half of that information was used at the follow-up visit. ${ }^{31}$ Weak and ineffective HIS management and inadequate NCD information, such as a lack of population-based NCD-related data on risk factors, mortality, disability and referral systems at the primary healthcare level, have been identified as crucial barriers to managing NCDs in Vietnam. ${ }^{41} 45$

\section{Leadership and governance}

Four studies investigated issues of leadership and stewardship in the management of NCDs in primary healthcare. ${ }^{41} 424547$ The research reported a lack of coordination among stakeholders and departments in implementing nationally designed NCD programmes/ interventions. A qualitative study in India discovered weak interdepartmental coordination between various government departments (eg, mental health programme and tobacco control programme), which resulted in poor NCD outcomes at the primary care level. ${ }^{42}$ The primary 
care-level NCD managers lacked knowledge of Vietnam's national NCD strategy or policies affecting targeted interventions for cancer, CVDs and diabetes. ${ }^{41}$ Limited knowledge of NCD management strategy and insufficient leadership capacity were highlighted among frontline healthcare staff. ${ }^{41}$ Furthermore, a lack of interaction between private and public providers and stakeholders was reported for NCD prevention/management activities in Vietnam. ${ }^{45}$ A mixed-method study found that Vietnam's nationally targeted NCD management and control programme lacked L\&G capacity. ${ }^{47}$

\section{Community perspective}

Only two studies, conducted in the Netherlands and New Zealand, explored community perspectives on patients' capacity for using healthcare information, selfmanagement and sharing problems when seeking aid to manage NCDs at the primary healthcare level. A mixedmethod study in the Netherlands ${ }^{46}$ showed that, during a consultation, people with diabetes had a low-level ability to share psychological issues with healthcare providers at the primary healthcare level. In New Zealand, the readiness of patients with NCDs (cancer, chronic pain, diabetes and mental health problems) was low, with only $36 \%$ of them seeking health-related information from digitalised sources. ${ }^{28}$ This demand-side perspective was not addressed in studies from LMICs.

\section{Quality of included studies/quality assessment}

Nearly three-fifth $(61 \%)$ of the studies were of good quality (MMAT score of 75) (table 1): 1 paper (4\%) had an MMAT score of 25 (low quality), 8 (35\%) scored 50 (medium quality), 11 (48\%) received 75 (good quality) and $3(13 \%)$ reached 100 (high quality). No study had an MMAT score of 0 (poor quality).

\section{DISCUSSION}

This review appraised available evidence on health system readiness for NCDs at the primary healthcare level. The key findings of this study were that health systems at the primary healthcare level were inadequately prepared for NCD prevention and management, and that readiness was poorly understood. Health system readiness was examined from the providers' perspectives, which is specifically focussed on the availability of infrastructures and supply of resources (eg, medicine, basic amenities, MPK\&T) as devised in the WHO SARA methodology or WHO PEN interventions. This may have narrowed the 'systems thinking' approach, which is a core philosophical basis that incorporates various elements and their interactions and interconnectedness to function as a system. ${ }^{19}$ Viewing the health system from this constricted sense categorically failed to include people's (service users') dimensions, which is an essential consideration for a well-functioning and inclusive health system. One plausible reason for predominantly analysing the health system from the supply-side perspective was the widespread acceptance of the WHO health system framework and its broader applications in individual studies. Over the past years, the 'building block' approach appeared as a dominant health system method globally, ${ }^{49}$ supporting the existing trend of assessing the health system from the supply-side perspective. Thus, the demand-side perspectives of health system readiness for NCDs warrant extensive investigation. Future research may focus on the demand-side aspects of the health system's readiness, such as community characteristics and associated determinants needed to establish an effective and inclusive health system to respond to the NCD epidemic.

This review demonstrated that almost all countries' primary healthcare systems have suffered from inadequate supply-side responses to medicine, technologies, equipment, amenities, trained healthcare professionals, health information and leadership and stewardship. The ill-equipped health system may result from insufficient financing mobilised through international and domestic channels and a lack of policy priority in responding to NCDs ${ }^{50-52}$ Among the NCDs addressed by the studies in this review, DM and HTN received the most attention in the current literature. Hence, other major NCDs such as CVD, CRD and cancer, which are prioritised by the WHO, remain largely under-researched. The focus on DM and HTN may be due to multiple factors, including increasing prevalence and associated determinants/risk factors for other NCDs in LMICs, a nationwide vertical programme, individual-level professional capacity and greater resource mobilisation, ${ }^{53-55}$ all of which have facilitated DM and HTN care, management and research. Moreover, the integrated model for DM and HTN care has widely been considered in the LMICs that accelerated the provision of effective and equitable HSD at the primary healthcare level, which would have helped to address the rising burden of them with accessible, equitable and costeffective interventions. ${ }^{56-58}$ This review revealed that at the primary healthcare level, health system readiness for major NCDs was primarily concentrated on the diagnosis and treatment aspects. However, readiness for health promotion and preventive interventions, provision of palliative care, screening, identification of risk factors, self-management and health education have remained underinvestigated and of less priority. ${ }^{59}$ As such, primary and secondary prevention of NCDs was emphasised in the WHO's NCD prevention and control strategy in $2011^{61}$ and has been highlighted in the current literature to reduce NCD-related morbidities and deaths. ${ }^{62-64}$ Preventive and health promotional activities on key NCD risk factors, ${ }^{6165}$ such as tobacco consumption, salt intake, physical inactivity, harmful alcohol use and unhealthy diet, stress that these can be addressed at the primary healthcare level to improve NCD outcome. The potential for a well-prepared health system is realised when promotional and preventive services are adequately provided at the primary healthcare level. ${ }^{667}$ Lack of a comprehensive prevention and management approach led us to hypothesise that the full potential of the health system's response 
to NCDs may have been hindered at the primary healthcare level. Majority of the studies in this review had good or high quality. However, a large proportion of the study reflected inexplicit evidence due to the methodology, small sample size, bias and incomplete information. A few quantitative studies lacked sufficient details about the participants' selection criteria, standard criteria for minimising bias and use of non-validated questionnaires with a relatively small sample size that might affect the scope of generalisability of the findings. ${ }^{27} 29323435$ One mixedmethod study was rated low quality due to the homogeneous sample and insufficient information about the data analysis. ${ }^{47}$ The rest of the mixed-method studies included in the review had a more representative sample size and methodological rigours. The majority of the included studies used the WHO's health system framework as an analytical basis to identify the health system components. However, some studies lacked a deeper analysis of the interplay and interconnectedness between different health system components. Despite these limitations, this study provides important information regarding current evidence on the readiness of the primary healthcare system for NCDs. Additionally, most of the selected studies in this review were conducted in resource-poor settings, primarily in sub-Saharan African and South East Asian countries. The smaller number of studies in developed countries may be explained by their adoption of a specialised disease management strategy, which lessens the focus on comprehensive management of NCDs at the primary healthcare level. ${ }^{68}$ An extensive investigation of community characteristics and associated factors may be necessary for establishing a well-functioning and more responsive health system to respond to NCDs. ${ }^{24}$

\section{Strengths and limitations}

This review's main strength was an inclusive data synthesis informed by the health system dynamics framework, which offers a deeper and more comprehensive (both supplyside and demand-side factors) understanding of primary healthcare system readiness for NCDs. Conducting an extensive systematic search of literature with handsearching references and expert advice increased the validity and trustworthiness of this review's findings. On the other hand, one of its limitations was that a few studies that reported health system readiness at combined primary and secondary healthcare levels were excluded. Moreover, the selected studies had heterogeneous study designs, methods and techniques, and focussed on a variety of health system components, preventing metaanalysis. Another limitation was that studies containing relevant information published in languages other than English have been excepted, which may have influenced the results of this review.

\section{CONCLUSION AND FUTURE DIRECTION}

This review demonstrated that health systems at the primary healthcare level are insufficiently prepared for
NCD prevention and management, especially for CVD, CRD and cancer. The existing health system response was characterised by insufficient 'supply-side' factors (ie, supply of medicine, equipment and technology), a lack of appropriate NCD management strategies and guidelines, a weak HIS, limited resources, uncoordinated local-level stewardship and leadership and a shortage of human resources. One of the notable findings was that the primary healthcare system's readiness over the years was evaluated from the 'supply-side' perspective; hence, there is a significant knowledge gap in the literature from the 'demand-side' standpoint. This observation may be useful for future research into users' views on NCD management at the primary healthcare level, including NCD management practice, knowledge, attitude, care-seeking behaviour, adherence to treatment, self-management and coping strategies.

\section{Twitter Md Nazmul Karim @Karim}

Contributors AK, MNK and BB created the manuscript. AK and LR led the literature search. AK, MNK, RMI and BB screened the literature and completed the mapping. AK led the drafting process, while MNK, RMI and BB provided substantial input. All authors read and approved the final manuscript. AK is the responsible author for overall content as guarantor.

Funding The authors have not declared a specific grant for this research from any funding agency in the public, commercial or not-for-profit sectors.

Competing interests None declared.

Patient consent for publication Not applicable.

Ethics approval This study does not involve human participants. This review has been done as part of a PhD project. The project has been approved by the Monash University Human Research Ethics Committee (Project ID: 27112) and Bangladesh Medical Research Council (Ref: BMRC/NREC/2019-2022/270).

Provenance and peer review Not commissioned; externally peer reviewed.

Data availability statement Data sharing not applicable as no datasets generated and/or analysed for this study. No data are available.

Supplemental material This content has been supplied by the author(s). It has not been vetted by BMJ Publishing Group Limited (BMJ) and may not have been peer-reviewed. Any opinions or recommendations discussed are solely those of the author(s) and are not endorsed by BMJ. BMJ disclaims all liability and responsibility arising from any reliance placed on the content. Where the content includes any translated material, BMJ does not warrant the accuracy and reliability of the translations (including but not limited to local regulations, clinical guidelines, terminology, drug names and drug dosages), and is not responsible for any error and/or omissions arising from translation and adaptation or otherwise.

Open access This is an open access article distributed in accordance with the Creative Commons Attribution Non Commercial (CC BY-NC 4.0) license, which permits others to distribute, remix, adapt, build upon this work non-commercially, and license their derivative works on different terms, provided the original work is properly cited, appropriate credit is given, any changes made indicated, and the use is non-commercial. See: http://creativecommons.org/licenses/by-nc/4.0/.

ORCID iDs

Ashraful Kabir http://orcid.org/0000-0002-5597-6065

Rakibul M Islam http://orcid.org/0000-0002-0889-8658

\section{REFERENCES}

1 World Health Organization. Noncommunicable diseases: key facts, 2021. Available: https://www.who.int/news-room/fact-sheets/detail/ noncommunicable-diseases

2 Marrero SL, Bloom DE, Adashi EY. Noncommunicable diseases: a global health crisis in a new world order. JAMA 2012;307:2037-8.

3 Beaglehole R, Epping-Jordan J, Patel V, et al. Improving the prevention and management of chronic disease in low-income and 
middle-income countries: a priority for primary health care. Lancet 2008;372:940-9.

4 Capizzi S, De Waure C, Boccia S. Global burden and health trends of non-communicable diseases. A systematic review of key issues in public health. Springer, 2015: 19-32.

5 Bloom DE, Cafiero E, Jané-Llopis E. The global economic burden of noncommunicable diseases. program on the global demography of aging, 2012. https://www3.weforum.org/docs/WEF_Harvard_HE_ GlobalEconomicBurdenNonCommunicableDiseases 2011.pdf

6 Assembly UG. High level meeting on prevention and control of non-communicable diseases: political Declaration of the high-level meeting of the general assembly on the prevention and control of non-communicable diseases. DocumentA/66/L. 1. New York, NY: United Nations General Assembly, 2016.

7 World Health Organization. Global action plan for the prevention and control of noncommunicable diseases 2013-2020, 2013. Available: https://www.who.int/publications/i/item/9789241506236

8 World Health Organization. World health statistics 2016: monitoring health for the SDGs sustainable development goals, 2016. Available: https://www.google.com/search?q=World $\% 20 \mathrm{Health} \%$ 200rganization.\%20World\%20health\%20statistics\%202016:\% 20 monitoring\%20health\%20for\%20the\%20SDGs\%20sustainable\% 20development\%20goals:\%20World\%20Health\%20organization\% 202016.

9 NCD Countdown. Ncd countdown 2030: pathways to achieving sustainable development goal target 3.4. Lancet 2020;396.

10 Dodd R, Palagyi A, Jan S, et al. Organisation of primary health care systems in low- and middle-income countries: review of evidence on what works and why in the Asia-Pacific region. BMJ Glob Health 2019;4:e001487.

11 Rohde J, Cousens S, Chopra M, et al. 30 years after Alma-Ata: has primary health care worked in countries? Lancet 2008;372:950-61.

12 Organization WH. Primary health care: report of the International Conference on primary health care, Alma-Ata, USSR, 6-12 September 1978, 1978. Available: https://www.who.int/publications/ i/item/9241800011

13 Alvarez FN, Leys M, Mérida HER, et al. Primary health care research in Bolivia: systematic review and analysis. Health Policy Plan 2016;31:114-28.

14 Bitton A, Ratcliffe HL, Veillard JH, et al. Primary health care as a foundation for strengthening health systems in low- and middleincome countries. J Gen Intern Med 2017;32:566-71.

15 Dineen-Griffin S, Garcia-Cardenas V, Williams K, et al. Helping patients help themselves: a systematic review of self-management support strategies in primary health care practice. PLoS One 2019;14:e0220116.

16 Albelbeisi AH, Albelbeisi A, El Bilbeisi AH, et al. Public sector capacity to prevent and control of noncommunicable diseases in twelve low- and middle-income countries based on WHOPEN standards: a systematic review. Health Serv Insights 2021;14:117863292098623.

17 Kabir A, Karim MN, Billah B. Primary healthcare system readiness to prevent and manage non-communicable diseases in Bangladesh: a mixed-method study protocol. BMJ Open 2021;11:e051961.

18 Organization WH. Everybody's business-strengthening health systems to improve health outcomes: WHO's framework for action, 2007. Available: https://apps.who.int/iris/handle/10665/43918

19 Bart C, Pirad M ON, Van der Vennet J. Basic concepts in public health. Antwerp: Institute of Tropical Medicine, 2013.

20 Mounier-Jack S, Griffiths UK, Closser S, et al. Measuring the health systems impact of disease control programmes: a critical reflection on the who building blocks framework. BMC Public Health $2014 ; 14: 278$.

21 Van Olmen J, Criel B, Van Damme W. Analysing health systems dynamics A framework. Studies in Health Services Organisation \& Policy. 28, 2012.

22 Moher D, Liberati A, Tetzlaff J, et al. Preferred reporting items for systematic reviews and meta-analyses: the PRISMA statement. PLoS Med 2009;6:e1000097.

23 Hong QN, Pluye P, bregues S F. Mixed methods appraisal tool (MMAT), version 2018, 2018: 10. http://mixedmethodsappraisaltoo Ipublic.pbworks.com/w/file/fetch/127916259/MMAT_2018 criteriamanual 2018-08-01_ENG.pdf

24 Olmen JV, Criel B, Bhojani U, et al. The health system dynamics framework: the introduction of an analytical model for health system analysis and its application to two case-studies. Health, Culture and Society 2012;2:1-21

25 Adinan J, Manongi R, Temu GA, et al. Preparedness of health facilities in managing hypertension \& diabetes mellitus in Kilimanjaro, Tanzania: a cross sectional study. BMC Health Serv Res 2019;19:537.
26 Bintabara D, Mpondo BCT. Preparedness of lower-level health facilities and the associated factors for the outpatient primary care of hypertension: evidence from Tanzanian national survey. PLoS One 2018;13:e0192942.

27 Duong DB, Minh HV, Ngo LH, et al. Readiness, availability and utilization of rural Vietnamese health facilities for community based primary care of non-communicable diseases: a CrossSectional survey of 3 provinces in northern Vietnam. Int $J$ Health Policy Manag 2019;8:150-7.

28 Honey M, Waterworth S, Aung $\mathrm{H}$. Older consumers' readiness for ehealth in New Zealand. Stud Health Technol Inform 2016;225:178-82.

29 Islam MR, Laskar SP, Macer D. A study on service availability and readiness assessment of non-communicable diseases using the who tool for Gazipur district in Bangladesh. Bangladesh Journal of Bioethics 2016;7:1-13.

30 Katende D, Mutungi G, Baisley K, et al. Readiness of Ugandan health services for the management of outpatients with chronic diseases. Trop Med Int Health 2015;20:1385-95.

31 Mendis S, Al Bashir I, Dissanayake L, et al. Gaps in capacity in primary care in low-resource settings for implementation of essential noncommunicable disease interventions. Int J Hypertens 2012;2012:1-7.

32 Musinguzi G, Bastiaens $\mathrm{H}$, Wanyenze RK, et al. Capacity of health facilities to manage hypertension in Mukono and Buikwe districts in Uganda: challenges and recommendations. PLoS One;10:e0142312.

33 Mutale W, Bosomprah S, Shankalala P, et al. Assessing capacity and readiness to manage NCDS in primary care setting: gaps and opportunities based on adapted who pen tool in Zambia. PLoS One;13:e0200994.

34 Nyarko KM, Ameme DK, Ocansey D, et al. Capacity assessment of selected health care facilities for the pilot implementation of package for essential non-communicable diseases (pen) intervention in Ghana. Pan Afr Med J 2016;25:16.

35 Pakhare A, Kumar S, Goyal S, et al. Assessment of primary care facilities for cardiovascular disease preparedness in Madhya Pradesh, India. BMC Health Serv Res 2015;15:408.

36 Peck R, Mghamba J, Vanobberghen F, et al. Preparedness of Tanzanian health facilities for outpatient primary care of hypertension and diabetes: a cross-sectional survey. Lancet Glob Health 2014;2:e285-92

37 Volk RJ, Foxhall LE. Readiness of primary care clinicians to implement lung cancer screening programs. Prev Med Rep 2015;2:717-9.

38 Biswas T, Haider MM, Das Gupta R, et al. Assessing the readiness of health facilities for diabetes and cardiovascular services in Bangladesh: a cross-sectional survey. BMJ Open 2018;8:e022817.

39 National Institute of Population Research and Training. Bangladesh health facility survey: 2017, 2020.

40 Aekplakorn W, Suriyawongpaisal P, Sirirassamee B. Assessment of capacity for cardiovascular disease control and prevention in Thailand: a qualitative study. Southeast Asian Journal of Tropical Medicine \& Public Health;36:741-7.

41 Kien VD, Van Minh H, Giang KB, et al. Views by health professionals on the responsiveness of commune health stations regarding noncommunicable diseases in urban Hanoi, Vietnam: a qualitative study. BMC Health Serv Res 2018;18:392.

42 Panda R, Mahapatra S, Persai D. Health system preparedness in noncommunicable diseases: findings from two states Odisha and Kerala in India. J Family Med Prim Care 2018;7:565-70.

43 Elias MA, Pati MK, Aivalli $\mathrm{P}$, et al. Preparedness for delivering noncommunicable disease services in primary care: access to medicines for diabetes and hypertension in a district in South India. BMJ Glob Health 2017;2:e000519.

44 Meiqari L, Nguyen T-P-L, Essink D, et al. Strengthening human and physical infrastructure of primary healthcare settings to deliver hypertension care in Vietnam: a mixed-methods comparison of two provinces. Health Policy Plan 2020;35:918-30.

45 Thi Thuy Nga N, Thi My Anh B, Nguyen Ngoc N, et al. Capacity of commune health stations in chi Linh district, HAl Duong Province, for prevention and control of noncommunicable diseases. Asia Pac J Public Health 2017;29:94S-101.

46 van Dijk-de Vries A, van Bokhoven MA, de Jong S, et al. Patients readiness to receive psychosocial care during nurse-led routine diabetes consultations in primary care: a mixed methods study. Int $J$ Nurs Stud 2016;63:58-64.

47 Van Minh H, Do YK, Bautista MAC, et al. Describing the primary care system capacity for the prevention and management of noncommunicable diseases in rural Vietnam. Int $J$ Health Plann Manage 2014;29:e159-73.

48 Bawazir AA, Al-Surimi K, Suwaidan SD, et al. Capacity and readiness of primary health care centers for implementation of the basic 
strategy for prevention and control of non-communicable diseases in Saudi Arabia. A case study from the Ministry of national GuardHealth Affairs, Riyadh, Saudi Arabia. Saudi Med J 2019;40:614-8.

49 Sacks E, Morrow M, Story WT, et al. Beyond the building blocks: integrating community roles into health systems frameworks to achieve health for all. BMJ Glob Health 2018;3:e001384.

50 Allen LN. Financing national non-communicable disease responses. Glob Health Action 2017;10:1326687.

51 Mendis S. The policy agenda for prevention and control of noncommunicable diseases. Br Med Bull 2010;96:23-43.

52 Robinson HM, Hort K. Non-Communicable diseases and health systems reform in low-and-middle-income countries. Pac Health Dialog 2012;18:179-90.

53 Fonseca VA, Kirkman MS, Darsow T, et al. The American diabetes association diabetes research perspective. Diabetes Care 2012;35:1380-7.

54 Lastra G, Syed S, Kurukulasuriya LR, et al. Type 2 diabetes mellitus and hypertension: an update. Endocrinol Metab Clin North Am 2014;43:103-22.

55 Malekzadeh A, Michels K, Wolfman C, et al. Strengthening research capacity in LMICs to address the global ncd burden. Glob Health Action 2020;13:1846904.

56 Shigayeva A, Atun R, McKee M, et al. Health systems, communicable diseases and integration. Health Policy Plan 2010;25 Suppl 1:i4-20.

57 Nigatu T. Integration of HIV and noncommunicable diseases in health care delivery in low- and middle-income countries. Prev Chronic Dis 2012;9:E93.
58 Esterson YB, Carey M, Piette JD, et al. A systematic review of innovative diabetes care models in low-and middle-income countries (LMICs). J Health Care Poor Underserved 2014;25:72-93.

59 Gillam S. Is the Declaration of Alma Ata still relevant to primary health care? BMJ 2008;336:536-8.

60 Maciocco G. [Alma Ata 30 years on. Evolution and perspectives of primary health care]. Ann Ig 2008;20:389-99.

61 Mamudu HM, Yang JS, Novotny TE. Un resolution on the prevention and control of non-communicable diseases: an opportunity for global action. Glob Public Health 2011;6:347-53.

62 Ezzati M, Obermeyer Z, Tzoulaki I, et al. Contributions of risk factors and medical care to cardiovascular mortality trends. Nat Rev Cardiol 2015;12:508-30.

63 Kontis V, Bennett JE, Mathers CD, et al. Future life expectancy in 35 industrialised countries: projections with a Bayesian model ensemble. Lancet 2017;389:1323-35.

64 Lloyd-Sherlock PG, Ebrahim S, McKee M, et al. Institutional ageism in global health policy. BMJ 2016;354:i4514.

65 World Health Organization. A global review of primary health care: emerging messages: global report, 2003.

66 Jeet G, Thakur JS, Prinja S, et al. Community health workers for non-communicable diseases prevention and control in developing countries: evidence and implications. PLoS One 2017;12:e0180640.

67 World Health Organization. Preventing noncommunicable diseases. Available: https://www.who.int/activities/preventingnoncommunicable-diseases

68 Van Lerberghe W. The world health report 2008: primary health care: now more than ever: World Health organization, 2008. Available: https://www.who.int/whr/2008/whr08_en.pdf 